\title{
Effects of treatment on the histopathology of leprosy
}

\author{
I A Cree, G Coghill, A M C Subedi, N C Abbot, S R Butlin, P D Samson, \\ J Swanson Beck
} of Ophthalmology,

University of London,

Bath Street,

I A Cree

Department of Pathology, University of Dundee, Ninewells Hospital and Medical School, Dundee

G Coghill

A M C Subedi

N C Abbot

Anandaban Leprosy Hospital, Kathmandu, Nepal

S R Butlin

Richardson Leprosy

Hospital, Miraj,

District Sangli,

Maharashtra, India

P D Samson

International

Medical College,

Jalan Selangor,

Petaling Jaya,

Selangor

Darul Ehsan,

Kuala Lumpur,

Malaysia

J S Beck

Correspondence to:

Dr I A Cree.

Accepted for publication

25 August 1994
Department of

Pathology, Institute

London ECIV 9EL

\begin{abstract}
Aims-To identify the histological changes in leprosy skin lesions over the first few weeks after the start of leprosy treatment and to examine their relationship to reversal reaction.

Methods-Sequential skin biopsy during treatment with multiple drug therapy. In this study, a series of 28 patients was studied, from whom two or more biopsies were taken at two week intervals. Fourteen patients had paucibacillary leprosy (PBL) and 14 had multibacillary leprosy (MBL). Results-In most cases, granuloma fraction and bacterial index fell during treatment, the bacterial index being less sensitive than the granuloma fraction. Since the biopsies were fixed in buffered formalin and processed through to paraffin wax, little immunohistochemistry was feasible. However, there was strong evidence of immune activation, with increased expression of HLA-DR in the granulomas of MBL and PBL cases: the epidermis also expressed HLA-DR in several patients. Such changes may reflect $\gamma$ IFN production from granuloma lymphocytes. Patients with reversal reaction often showed HLA-DR expression on admission which decreased with corticosteroid treatment.
\end{abstract}

Conclusions-The results suggest that activation of cell mediated immunity in leprosy lesions occurs during treatment with multiple drug therapy and may not be restricted to those with clinical evidence of reversal reaction.

(f Clin Pathol 1995;48:304-307)

Keywords: Leprosy, reaction, antibiotics, HLA-DR, granuloma, histology, immunohistology.

Multiple drug therapy has revolutionised the treatment of leprosy and many patients are now cured within six months to two years, depending on their bacterial load. Recent developments in chemotherapy make it likely that

Table 1 Classification of patients studied on the Ridley-fopling scale

\begin{tabular}{lllllllr}
\hline & $I d t$ & $T T$ & $B T$ & $B B$ & $B L$ & $L L$ & Total \\
\hline Newly diagnosed & 4 & 1 & 8 & 0 & 6 & 3 & 22 \\
Reversal reaction & 0 & 0 & 1 & 0 & 3 & 2 & 6 \\
Total & 4 & 1 & 9 & 0 & 9 & 5 & 28 \\
\hline
\end{tabular}

$\mathrm{Idt}=$ Indeterminate $; \mathrm{TT}=$ tuberculoid $; \mathrm{BT}=$ borderline-tuberculoid $; \mathrm{BB}=$ borderline $; \mathrm{BL}=$ borderline lepromatous; $\mathrm{LL}=$ lepromatous. the period of treatment will be further reduced. However, patients often suffer immunologically mediated reactions, either spontaneously or during and after treatment. Two major types of reaction are recognised in type I, reversal or upgrading reaction, the skin becomes red and swollen with tenderness of peripheral nerves. This neuritis leads to additional disability. Type I reactions occur across the clinicopathological spectrum of disease from tuberculoid (few bacilli with epithelioid granulomas) to lepromatous leprosy (diffuse infiltration of macrophages containing enormous numbers of bacilli). Their association with "reversal" or "upgrading" of previously lowered resistance to Mycobacterium leprae is responsible for the name. In Type II reactions (erythema nodosum leprosum) painful inflamed nodules occur in the skin of lepromatous patients. These appear to be immune complex mediated.

Since reversal reaction probably results from increased cell mediated immunity to the leprosy bacillus following antigen release after the start of treatment, ${ }^{1}$ we have looked at the histological changes which occur in the first week weeks after starting multiple drug therapy. Histologically, reversal reaction is associated with an influx of lymphocytes and macrophage maturation, with the appearance of giant cells. The granuloma fraction increases in many patients. However, histological and clinical evidence of reaction often correlate poorly. ${ }^{23}$ We therefore looked at immunohistochemical indices as well as morphological features which might signal incipient reaction.

\section{Methods}

BIOPSIES

Skin punch biopsies $(4 \mathrm{~mm})$ were taken before and at least once after multiple drug therapy was started in 22 patients with leprosy diagnosed clinically by an experienced leprologist and confirmed in the first biopsy by histopathology (table 1). Patients in this group were either newly diagnosed (16 cases) or relapsed following partial treatment with dapsone monotherapy (six cases). In addition, two or more biopsies were taken before and during corticosteroid treatment of reversal reaction in six patients diagnosed clinically as being in reaction. Of the 28 cases, six were female and 22 male, with an age range of 16 to 65 years (mean 39 years). In all cases, biopsies were taken from the edge of the same large skin lesion to ensure reproducibility of histology. ${ }^{4}$ The biopsies were fixed in $4 \%$ buffered form- 
Table 2 Granuloma fraction results for all patients included in the study. In keeping with previous work, ${ }^{4}$ change (up or down) was defined as $>5 \%$ of dermal area

\begin{tabular}{lll}
\hline & Newly diagnosed & Reversal reaction \\
\hline Decrease & 15 & 6 \\
No change $(<5 \%)$ & 4 & 0 \\
Increase & 3 & 0 \\
\hline
\end{tabular}

aldehyde and processed in Dundee through to paraffin wax in a VIP2 vacuum processor with postfixation in phenol formalin. ${ }^{5}$ Sections were cut at $5 \mu \mathrm{m}$ for histology (haematoxylin and eosin), assessment of bacterial index (WadeFite), and immunohistochemistry.
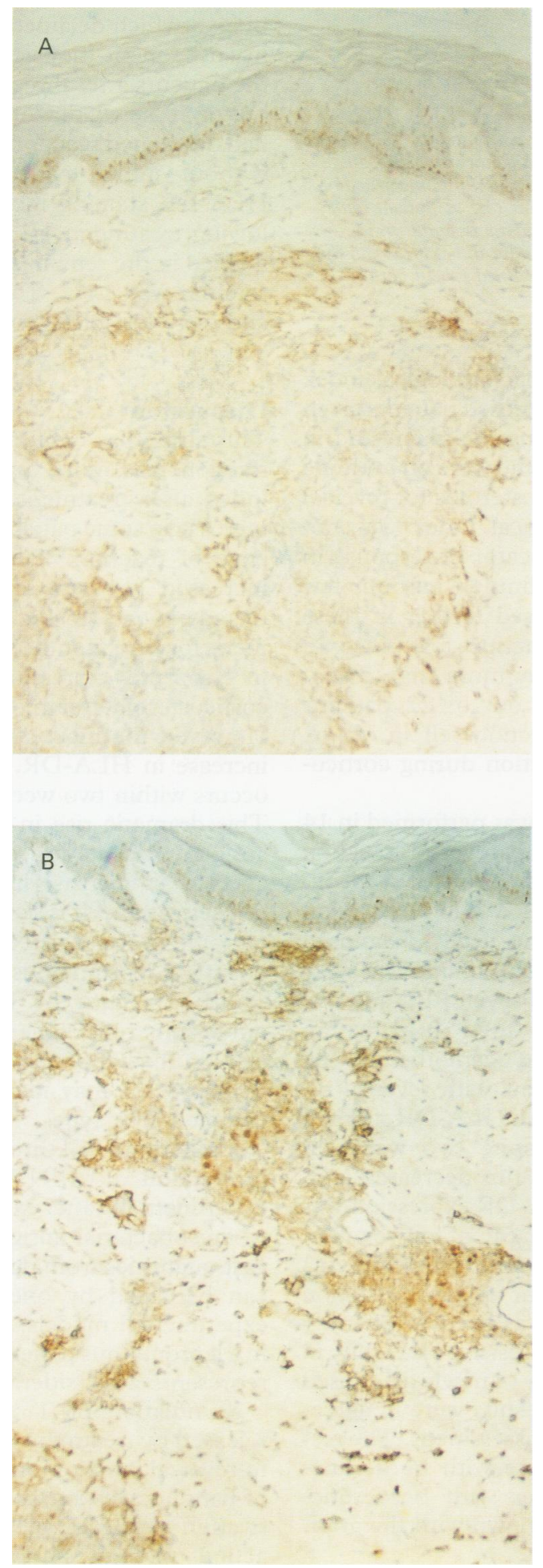

IMMUNOHISTOCHEMISTRY

Sections from each case were stained simultaneously by the avidin-biotin complex method for lysozyme (Dako), and HLA-DR (LN3 antibody, ICN Flow, since both antibody preparations work well with paraffin sections. A mild haemalum counterstain was used to allow detection of unstained cells.

\section{QUANTITATION}

Granuloma fraction was measured by planimetry using an Imagan 2 planimeter (Kompira) as previously described. ${ }^{46}$ Image analysis of sections stained for HLA-DR was performed using the $\times 20$ objective of an Olympus CK2 microscope fitted with a monochrome CCD camera linked to a Viglen $486 \mathrm{DX} / 2$ microcomputer with an image processing board and running Global Lab Image software (Data Translation). Using the particle detection tool and a section from the pretreatment biopsy, an optical density threshold was set which allowed stained areas to be detected without detection of background or counterstained features. This threshold was used for all sections from that case to allow comparison of HLA-DR expression over time. The threshold was reset for each case and the results for each section expressed as the percentage of granuloma stained.

\section{Results}

The variables assessed in relation to treatment were bacteriological index (MBL patients only), granuloma fraction, HLA-DR, and lyso-

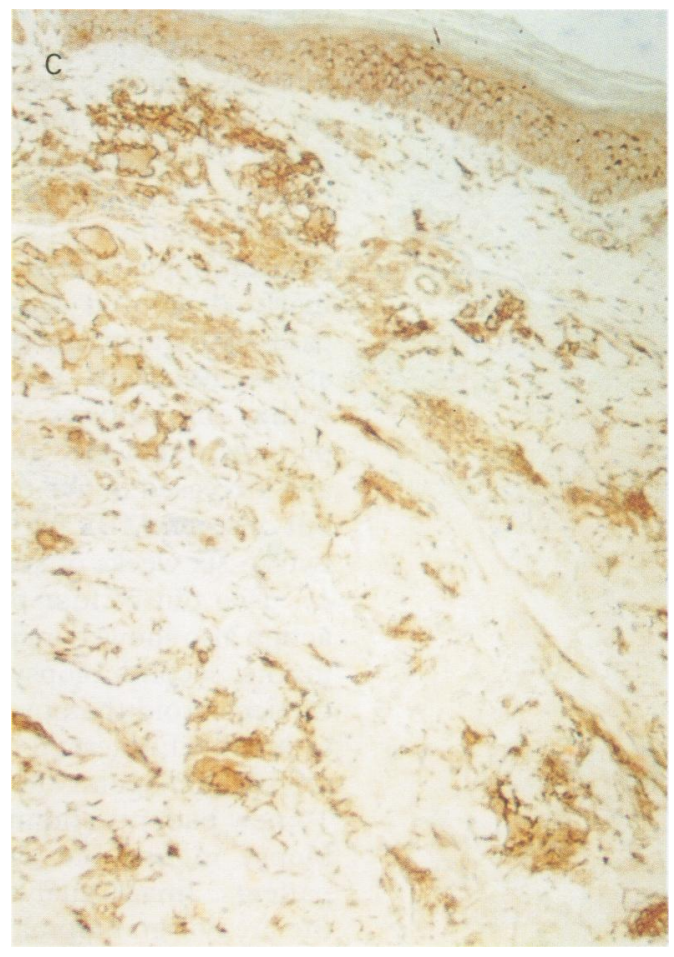

Figure 1 Change in HLA-DR staining over time in a patient with borderline-tuberculoid leprosy (bacteriological index $=1)$. The untreated biopsy $(A)$ shows a large granuloma with some $H L A-D R$ expression at its edge, while after two weeks (B) there is considerably increased $H L A-D R$ expression with positivity in giant cells. The third biopsy (C) taken after four weeks shows an even greater degree of expression with positivity of the epidermis. 

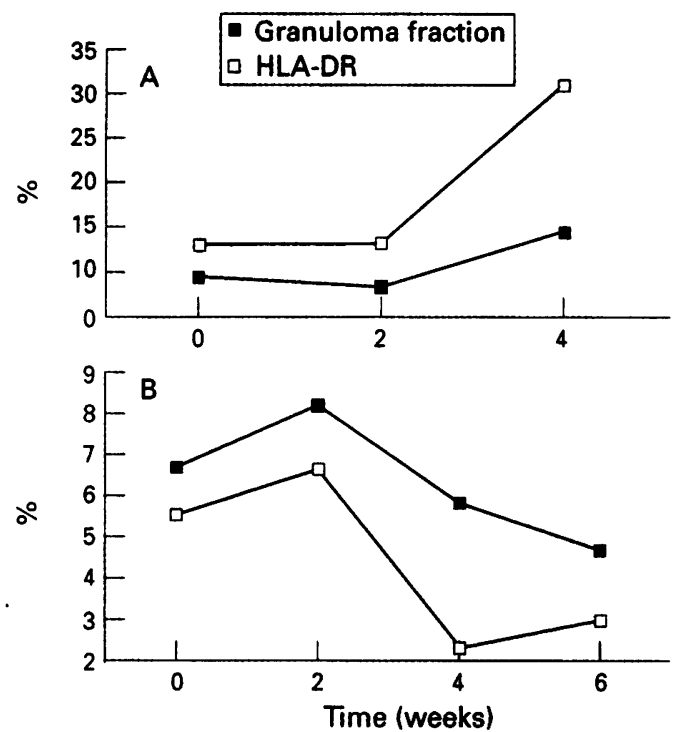

Figure 2 The results of image analysis of $H L A-D R$ expression in two previously untreated patients. (A) $A$ patient with borderline-tuberculoid leprosy showing an increase in $H L A-D R$ expression and granuloma fraction (GF) over four weeks of treatment. (B) A patient with borderline-lepromatous leprosy showing a small initial increase in both HLA-DR expression and GF during the first two weeks, followed by a sustained fall in both measurements.

zyme. In MBL patients, bacteriological index decreased in 3/9 newly diagnosed patients given one month or multiple drug treatment and in $2 / 5$ patients with reaction who were on multiple drug treatment with corticosteroids for reversal reaction. The bacteriological index was not found to be a reliable indicator of response in PBL patients, who by definition harbour few bacilli, and was not assessed further in these cases. The granuloma fraction fell in 15/22 biopsies, but was raised by more than $5 \%$ of the dermal area in three out of 22 patients (table 2). Granuloma fraction fell in all six patients with reversal reaction during corticosteroid treatment.

Immunohistochemistry was performed in 14 newly diagnosed and four reversal reaction cases with a granuloma fraction of $>4 \%$ to assess lysozyme and HLA-DR expression within the biopsies. In the newly diagnosed patients, HLA-DR immunostaining was visually increased in five out of seven PBL and five out of seven MBL patients (total 10/14) within two to six weeks of starting chemotherapy (fig 1). Two out of four patients with reversal reaction also showed increased HLA-DR staining in the second biopsy despite two weeks of corticosteroid therapy, but this decreased subsequently. Epidermal HLA-DR expression was seen at some point after treatment in five out of 14 new patients and in the first (pretreatment) biopsy in two out of four reversal reaction patients. Image analysis was used to quantify the area of granuloma expressing HLA-DR in batch stained sections from 14 newly diagnosed patients. In all cases the results were in agreement with previous visual assessment. The results from two patients, one with borderlinetuberculoid and the other with borderlinelepromatous leprosy, are shown with the granuloma fraction data in fig 2 .
Lysozyme was present to some degree in at least one biopsy in all patients except one with lepromatous leprosy. It was visually stronger in patients with tuberculoid/borderline-tuberculoid leprosy and reversal reaction. In the four reversed reaction patients on corticosteroids, lysozyme expression appeared to diminish with time, while in four of eight and two of six MBL newly diagnosed patients, it appeared to increase. Lysozyme staining was often patchy within the cell and was unsuitable for image analysis.

Three of the patients (two borderline-tuberculoid, one borderline lepromatous) were given prednisolone therapy on admission as they were thought to be in reaction: all three showed HLA-DR and lysozyme expression in their first biopsy, which declined or showed little change in subsequent biopsies. Clinical follow up of the patients after three years showed that three out of 20 newly diagnosed or relapsed patients had developed reactions, including two patients without initial evidence of reaction whose HLA-DR staining increased considerably following treatment; HLA staining was not performed in the remaining case as the granuloma fraction was $<4 \%$. Two patients moved away and were lost of follow up.

\section{Discussion}

This study has confirmed that the granuloma fraction falls more quickly than the bacterial index after treatment starts, and that it may increase in some patients with histological evidence of reaction. ${ }^{2}$ There is also evidence of increased lysozyme expression in newly detected patients treated with multiple drug treatment for one month, and of a rapid reduction in lysozyme expression in those patients on corticosteroid treatment for reversal reaction. However, the most striking observation is the increase in HLA-DR, which is some patients occurs within two weeks of starting treatment. This dramatic rise in expression of a class II antigen presentation molecule is strong evidence of an increase in macrophage/epitheloid cell activation. ${ }^{7}$ It is seen following the injection of $\gamma$-interferon ( $\gamma \mathrm{IFN}$ ) into lesions ${ }^{8}$ and exposure of lepromatous macrophages in culture to $\gamma \mathrm{IFN}$ or antigen. ${ }^{9}$ It is therefore likely that our results reflect the local production of this cytokine by lymphocytes within the granuloma. This process may involve CD $4+\mathrm{T}$ lymphocytes of Th1 types, NK cells, $\gamma \delta$ cells, or a combination of all three possibilities. Increased production of $\gamma \mathrm{IFN}$ by lymphocytes is seen experimentally after exposure of $T$ lymphocytes to mycobacterial antigen, which is released in vast quantities from dead bacilli, $99 \%$ of which can be killed by one dose of rifampicin in experimental models. Since the bacillary load in a lepromatous patient may be $10^{13}$ bacilli, this represents a considerable antigenic challenge.

Stimulation of $T$ lymphocytes by antigen released by treatment is widely believed to lead to reversal reaction, in which there is an influx of lymphocytes and increased macrophage activation with giant cell formation. Reversal reaction patients show granuloma HLA-DR 
expression on admission and we have shown that this decreases with steroid therapy. Expression of HLA-DR within a short time of starting multiple drug treatment may therefore be a good marker for the tendency to develop reversal reaction, although many tuberculoid cases show granuloma HLA-DR expression on admission. ${ }^{10-13}$ In this study, most of our patients developed increased HLA-DR expression, unless they were given corticosteroids, suggesting that activation of cell mediated immunity in leprosy lesions occurs in most patients and is not restricted to those with upgrading reaction. It has previously been reported that treatment increases cytokine expression within the lesion ${ }^{14}$ and serum soluble IL2 receptor concentrations. ${ }^{15}$ These findings suggest that the difference between patients who develop reversal reaction and those who do not is likely to be quantitative rather than qualitative, although this requires further investigation. ${ }^{3}$ Since silent neuritis and reversal reaction are known to occur in many patients, ${ }^{1617}$ it is possible that the reversal reaction represents a clinically identifiable degree of an immunological process which occurs in all patients treated for leprosy.

Larger studies are required to confirm these preliminary results, and to correlate them with immunological variables ${ }^{15}$ and markers of nerve or vascular function. ${ }^{1819}$ The ability to follow immunological changes within the granuloma quantitatively by sequential biopsy, immunohistochemistry, and image analysis should be considered as a tool for use in future clinical trials of modified multiple drug treatment regimens in leprosy.

We are grateful to LEPRA and the Leprosy Mission for their support and to the doctors, paramedical staff and patients of the Richardson Leprosy Hospital. We also wish to thank Drs G Kaplan, D Scollard, and S Lucas for their advice.

1 Cree IA, Smith WCS, Rees RJW, Beck JS. The influence of anti-myobacterial chemotherapy on delayed hyper- sensitivity skin-test reactions in leprosy patients. Lepr Rev 1988;59:145-51

2 Ridley DS. Skin biopsy in leprosy, 2nd edn. Basel: CibaGeigy, 1985

3 Mukherjee A, Cree IA, Abalos R, Chacko CJG, Desikan $\mathrm{KV}$, Fields JP, et al. 14th International Leprosy Congress - Pathology Workshop Report. Int F Lepr 1994;61:737-9.

4 Cree IA, Srinivasan T, Krishnan SAR, Gardiner CA, Mehta J, Fisher CAH, et al. Reproducibility of histology in leprosy J, Fisher CAH, et al. Reproducibility of
lesions. Int $\mathcal{F}$ Lepr 1988;56:296-301.

5 Slidders WS, Hopwood D. Buffered phenol formaldehyde (pH 7.0 and $\mathrm{pH} 5 \cdot 5$ ): improved fixation in an enclosed (pH $7 \cdot 0$ and $\mathrm{pH} 5 \cdot 5)$ : improved fixation in
tissue processor. Med Lab Sci 1989;46:74-5.

6 Cree IA, McDougall AC, Coghill G, Beck JS. Quantification of the granuloma fraction in leprosy skin biopsies by planimetry. Int $\mathcal{F}$ Lepr 1985;54:607-13.

7 Voc-Platzer B, Kremsner P, Stemberger H, Wiederman G. Restoration of defective cytokine activity within lepromatous leprosy lesions. Int $\mathcal{f}$ Med Microbiol 1990;272. 458-66.

8 Kaplan G, Nusrat A, Sarno EN, Job CK, McElrath J, Porto $\mathrm{JA}$, et al. Cellular responses to the intradermal injection of recombinant human gamma-interferon in lepromatous leprosy patients. Am $\mathcal{F}$ Pathol 1987;128:345-53.

9 Desai SD, Birdi TJ, Antia NH. Correlation between macrophage activation and bactericidal function and $M y c o-$ bacterium leprae antigen presentation in macrophages of leprosy patients and normal individuals. Infect Immun 1989;57:1311-7.

10 Ridley MJ, Ridley DS. Unique expression of HLA-DR (lalike) antigen in the lesions of polar tuberculoid leprosy. Lepr Rev 1982;53:249-52.

11 Modlin RL, Hofman FM, Meyer PR, Sharma OP, Taylor $\mathrm{CR}$, Rea TH. In situ demonstration of T lymphocyte subsets in granulomatous inflammation: leprosy, rhinoscleroma and sarcoidosis. Clin Exp Immunol 1983;51:4308 .

12 Narayanan RB, Bhutani LK, Sharma AK, Nath I. T cell subsets in leprosy lesions; in situ characterization using monoclonal antibody. Clin Exp Immunol 1983;51:421-9.

13 Collings LA, Tidman N, Poulter LW. Quantitation of HLADR expression by cells involved in the skin lesions of tuberculoid and lepromatous leprosy. Clin Exp Immunol 1985;61:58-66.

14 Arnoldi J, Gerdes J, Flad H-D. Immunologic assessment of cytokine production of infiltrating cells in various forms of leprosy. Am ₹ Pathol 1990,137:749-53.

15 Tung KSK, Umland E, Matzner P, Nelson K, Schauf V, Rubin L, et al. Soluble serum interleukin 2 receptor levels in leprosy patients. Clin Exp Immunol 1987;68:10-15.

in leprosy patients. Clin Exp Immunol 1987;68:10-15.
16 Job CK. Nerve damage in leprosy. Int F Lepr 1989;57:532-9.

17 Duncan ME, Melsom R, Pearson JMH, Ridley DS. The association of pregnancy and leprosy I. New cases, relapse of cured patients and deterioration of patients on treatment during pregnancy and lactation: results of a prospective study of 154 pregnancies in 147 Ethiopian women. Lepr Rev 1981;52:245-62.

18 Agusni I, Beck JS, Potts RC, Crea IA, Ilias MI. Blood flow velocity in the cutaneous lesions of leprosy. Int $\mathcal{F}$ Lepr 1988;56:394-400.

19 Beck JS, Abbot NC, Samson PD, Butlin CR, Grange JM, Cree IA, et al. Impairment of vasomotor reflexes in the fingertips of leprosy patients. $\mathcal{F}$ Neurol Neurosurg Psychiatr 1991;54:965-71. 